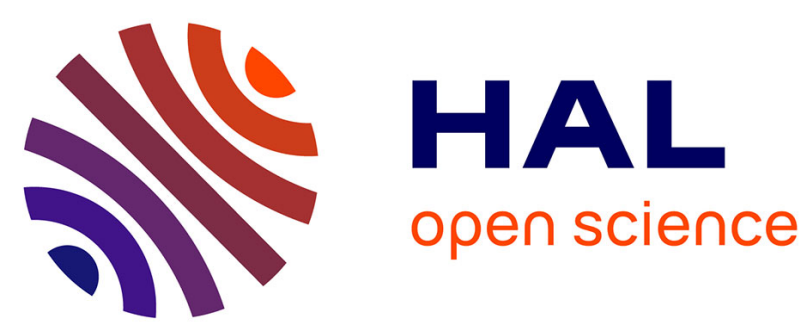

\title{
Thermal transport in semiconductors studied by Monte Carlo simulations combined with the Green-Kubo formalism
}

\author{
David Lacroix, Mykola Isaiev, Gilles Pernot
}

\section{- To cite this version:}

David Lacroix, Mykola Isaiev, Gilles Pernot. Thermal transport in semiconductors studied by Monte Carlo simulations combined with the Green-Kubo formalism. Physical Review B, 2021, 104 (16), pp.165202. 10.1103/physrevb.104.165202 . hal-03516582

\section{HAL Id: hal-03516582 \\ https://hal.univ-lorraine.fr/hal-03516582}

Submitted on 7 Jan 2022

HAL is a multi-disciplinary open access archive for the deposit and dissemination of scientific research documents, whether they are published or not. The documents may come from teaching and research institutions in France or abroad, or from public or private research centers.
L'archive ouverte pluridisciplinaire HAL, est destinée au dépôt et à la diffusion de documents scientifiques de niveau recherche, publiés ou non, émanant des établissements d'enseignement et de recherche français ou étrangers, des laboratoires publics ou privés. 


\title{
Thermal transport in semiconductors studied by Monte Carlo simulations combined with the Green-Kubo formalism
}

\author{
David Lacroix $\odot,{ }^{*}$ Mykola Isaiev $\odot$, and Gilles Pernot $\odot$ \\ Université de Lorraine, CNRS, LEMTA, Nancy F-54000, France
}

(Received 2 July 2021; revised 9 September 2021; accepted 5 October 2021; published 19 October 2021)

\begin{abstract}
In this paper, we demonstrate that it is possible to combine two computational approaches, usually used in very different contexts, namely, the Green-Kubo formalism and statistical approaches, to solve the Boltzmann transport equation to calculate the thermal conductivity of semiconductors. In this framework, first the phonon transport is solved by the Monte Carlo method according to the Debye-Callaway or Holland formalisms, then the flux autocorrelation is calculated. This new approach has been implemented to calculate the properties of $\mathrm{Si}, \mathrm{Ge}, \mathrm{GaN}$, and $\mathrm{C}$ (diamond) in an extended temperature range, from 50 to $600 \mathrm{~K}$. The simulation results are compared with those given by experimentation, with very good agreement.
\end{abstract}

DOI: 10.1103/PhysRevB.104.165202

\section{INTRODUCTION}

Thermal transport properties of semiconductor materials have been extensively studied since the early 1960s, with the continuous development of sciences related to electronics and their applications [1]: first, with more and more accurate experiments supported by theoretical modeling; then, more recently, with the increasing use of computational material science theories like density functional theory (DFT) and molecular dynamics (MD) at the atomistic level. Such approaches are now considered of utmost interest for seeking and characterizing tomorrow's materials, especially with the help of artificial intelligence techniques. In thermal sciences, semiconductors' most studied transport property is their "lattice thermal conductivity" (TC), which can be derived from the phonon propagation and scattering. This quantity can be computed using different approaches, at equilibrium or out of equilibrium, which are relevant at different scales, each having advantages and weaknesses. Among them, DFT combined with the solution of the Boltzmann transport equation (BTE) is known to provide accurate results with a few approximations $[2,3]$. Furthermore, nonequilibrium MD and equilibrium MD methods [4,5], based on the use of interatomic potential, allow calculation of the thermal properties of materials. In particular, equilibrium MD uses fluctuations of heat currents and correlations of the latter give, through the Green-Kubo (G-K) formalism, access to the TC of materials. This approach, unlike nonequilibrium MD, does not require the presence of thermostats and is therefore less sensitive to the system's geometry. However, all MD approaches remain limited to structures involving a few million atoms and should, in theory, be used for temperatures above the Debye one. At mesoscopic scales, solution of the BTE in the framework of the relaxation time approximation has also proved its ability to provide accurate estimation of the TC for bulk

\footnotetext{
*david.lacroix@univ-lorraine.fr
}

as well as for nanostructured materials. In this case, Monte Carlo (MC)-based simulations are well adapted to statistically mimic phonon transport under a thermal gradient and their intrinsic and boundary interactions [6]. These three methods are more and more closely related, as DFT can provide useful inputs like phonon lifetimes, potentials, dispersion properties, etc., for MD [7] and MC [8] methods.

In this context, there is room for new methods to compute a material's TC that can take advantage of the forces of the existing ones. Here, we report on the study of equilibrium heat flux fluctuations by MC and how to determine thermal transport properties via the Green-Kubo formula. In gases, very recent work by Bruno [9] shows that direct simulation Monte Carlo computations combined with the G-K formula allow one to recover fluid transport properties such as the diffusion coefficient, shear viscosity, and thermal conductivity. In solids, such an approach can also be used. Indeed, to the best of our knowledge, we provide here for the first time its derivation to semiconductor crystals like $\mathrm{Si}, \mathrm{Ge}, \mathrm{GaN}$, and $\mathrm{C}$ (diamond). In the following, we detail our methodology and provide comparisons between computed and measured TCs of these materials over a large temperature range. The phonon confinement effect and its major impact on nanostructured materials' TC are also discussed.

\section{PHONON DYNAMICS}

\section{A. Boltzmann transport equation for phonons}

Phonon dynamics is governed by the BTE. In the classic assumption of no external forces, it reads

$$
\frac{\partial f}{\partial t}+\nabla_{\mathbf{K}, p} \omega \cdot \nabla_{\mathbf{r}} f=\left.\frac{\partial f}{\partial t}\right|_{\mathrm{col}},
$$

with $f(\mathbf{K}, p, \mathbf{r}, t)$ the energy carrier distribution function. The left-hand side of Eq. (1) refers to phonon transport, while the right-hand side is related to phonon scattering. In the $\mathrm{MC}$ approach, phonons are considered particles, with a 


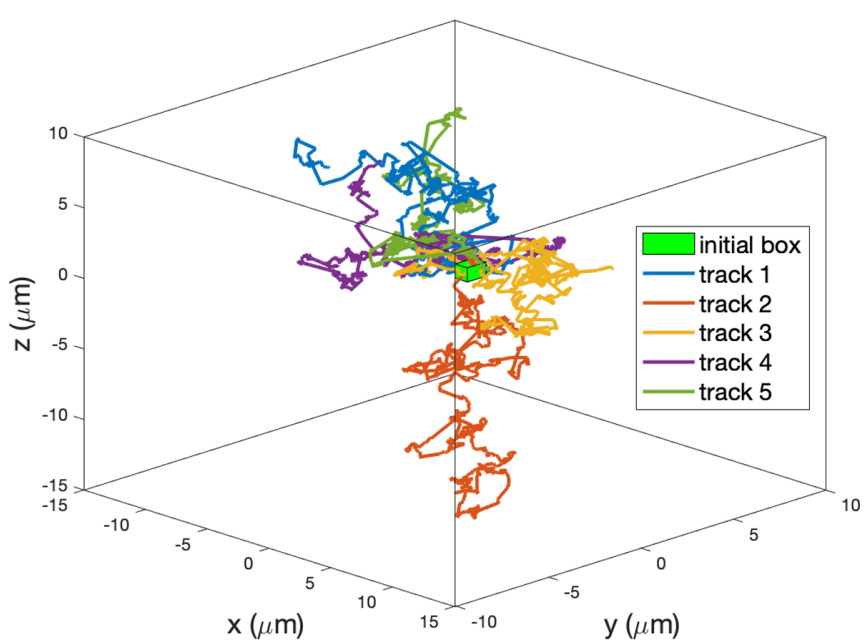

FIG. 1. Five phonon trajectories in bulk $\mathrm{Si}$ at $T=300 \mathrm{~K}$ after 100000 time steps $(\delta t=1 \mathrm{ps})$ without boundary scattering (bulk case). The initial particle's locations are within the green box $(V=1 \times 1 \times 1 \mu \mathrm{m})$.

given frequency and polarization, that travel according to their group velocity along different propagation directions. Phonon scattering involves three-phonon interactions and phononimpurity or -defect ones. In addition, boundary scattering can be considered in the case of nanostructure and thus also takes into account phonon mean free path reduction. "Normal," "Umklapp," and "impurity" scattering processes are here described in the frame of the relaxation time approximation, which allows us to linearize the right-hand side of Eq. (1). In the MC procedure, phonon scattering is addressed through the calculation of a collision probability, $P_{\text {col }}$, the latter being a function of the calculated phonon lifetime and simulation time step $\delta t$,

$$
P_{\mathrm{col}}(\omega, p, T)=1-\exp \left(\frac{-\delta t}{\tau_{\mathrm{tot}}(\omega, p, T)}\right),
$$

with $\tau_{\text {tot }}(\omega, p, T)$ the total relaxation time of the phonon mode $(\mathbf{K}, p)$. This relaxation time can be expressed according to the Matthiessen rule about individual relaxation time inverse summation. In this study, both the Debye-Callaway model [10] and the Holland model [11] were used to compute intrinsic phonon lifetimes considering isotropic dispersion properties for the four materials of interest, i.e., Si, Ge, GaN, and C. All these properties are reported in the Supplemental Material [12]). For a given initial phonon population, suitably sampled using the phonon cumulative distribution function [6] at a given temperature, it is possible to perform time iterative modeling of phonon transport in the frame of the MC approach and to collect simulation outputs such as particle trajectories and, of course, heat flux fluctuation along time. Hereafter are plotted five phonon tracks followed during $N_{t}=$ 100000 times steps of 1 ps (see Fig. 1) in the case of bulk $\mathrm{Si}$ at $300 \mathrm{~K}$. Usually, $N_{p}=500$ "particles" (phonon) trajectories are followed to ensure sufficient statistical sampling. Each modification of the trajectory corresponds to a collision process of the Umklapp or normal type or associated with an impurity. The number of required time steps depends on the mean free path (mfp) of the energy carriers. It increases

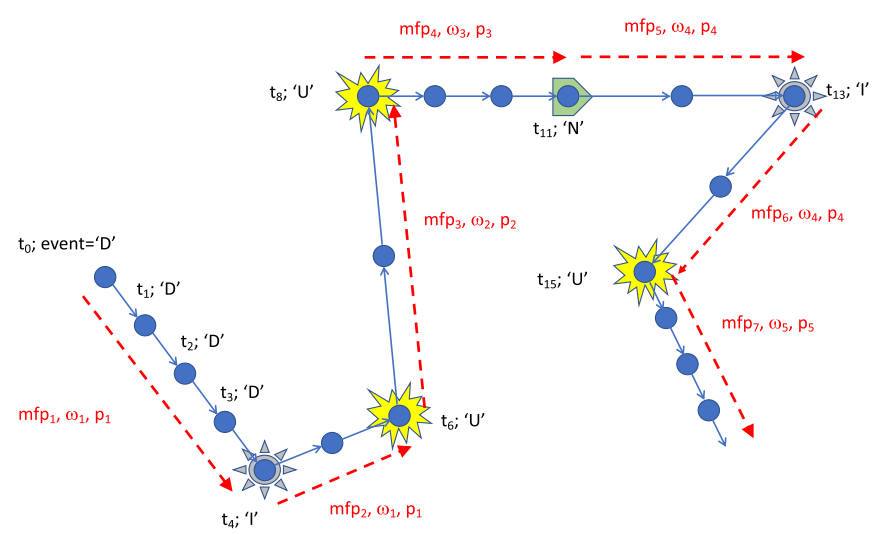

FIG. 2. Analysis of the particle mfp and phonon state during successive time steps of the simulation.

as the temperature decreases. Details about these issues are discussed in the paragraph related to the convergence of the results (see Sec. II D).

\section{B. Mean free path calculations}

For $\mathrm{Si}$ at $300 \mathrm{~K}$, phonon transport is in an intermediate regime between ballistic and diffusive behavior [13]. Therefore, preliminary investigations aim to identify mfp characteristics between scattering events. In this work, phonon mfp's are not directly derived from lifetime analysis assuming $\Lambda \simeq v_{g} \times \tau$. Here, a postprocessing analysis of the particle trajectories is carried out to compute the distance traveled by a phonon between two distinct scattering events (mfp's) and we relate this length to the current state of the phonon, i.e., its frequency $\omega$ and its polarization state LA or TA; the procedure is the following one.

Mean free path analysis was achieved by considering all the scattering events that occur during the $N_{t}$ time steps of a simulation (typically, $N_{t}=100000$ ). For this purpose, we have used 5 particles among 500 and calculated the length of phonon trajectories between each collision due to impurity, normal, or Umklapp processes. The applied procedure is as follows:

(i) First, we recall that at each time step in the simulation we keep in mind the phonon location $(x, y, z)$, its state (frequency, polarization), and the nature of the last scattering event, which can be no scattering (drift; D), impurity (I), normal (N), or Umklapp (U) scattering.

(ii) At the end of the MC calculation (particle tracking stage) mfp's are therefore obtained by computing the lengths of the particle trajectories between two scattering events, i.e., when the state skips from D to I or from $\mathrm{N}$ or U (see Fig. 2). In the Monte Carlo process, for I scattering, only the propagation direction is randomly redrawn. For $\mathrm{N}$ scattering, we conserve the propagation direction and redrawn phonon frequency and polarization and thus change the group velocity. For U scattering both the propagation direction and the phonon state are redrawn.

(iii) Thus, during postprocessing of the results we can count the number of phonon mfp's and identify for each of them the current frequency and polarization (LA or TA). 

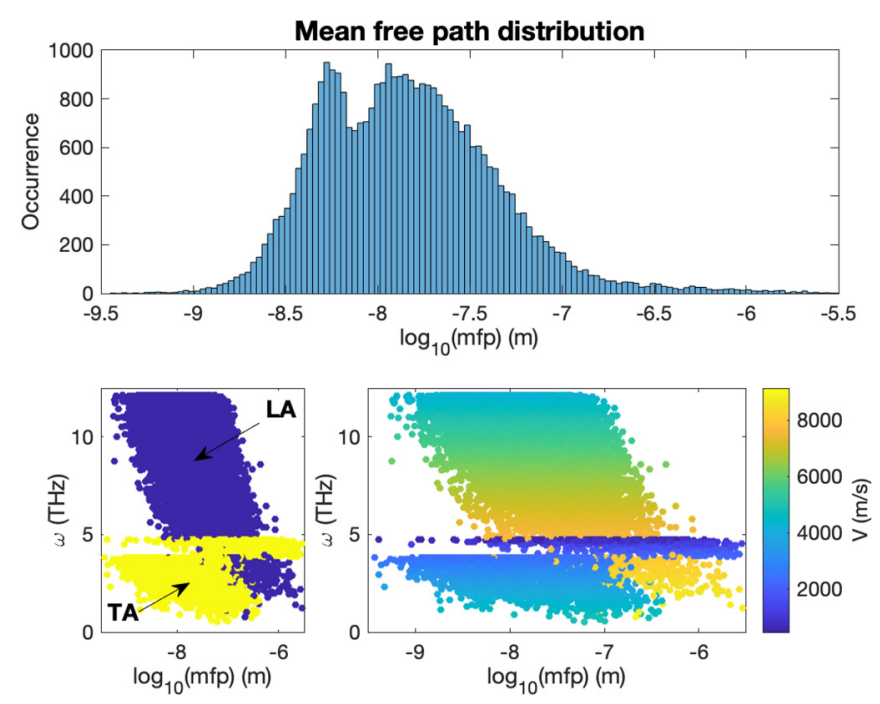

FIG. 3. Top: Phonon mfp distribution for Si at $300 \mathrm{~K}$. Bottom: Phonon $\mathrm{mfp}$ as a function of polarization, frequency, and group velocity. Five particles were followed during 100000 time steps of 1 ps. Phonon lifetimes according to Holland's formalism [11].

These mfp's are given in the histogram in Fig. 3 (150 bins in each of them); they are in the range of some nanometers to tens of micrometers (at low $T$ ). In addition, we report in the scatterplots the polarization, the frequency, and the group velocity (indexed color) of the followed particles (see Fig. 3). Typically, for $\mathrm{Si}$ at $300 \mathrm{~K}$, we count around 7000 distinct mfp's for one particle trajectory composed of 100000 time steps. In Fig. 1, five particles' trajectories are considered, leading to roughly $35000 \mathrm{mfp}$ 's.

The obtained results are consistent with the expected behavior of phonon transport. At low $T(100 \mathrm{~K})$, the $\mathrm{mfp}$ distribution is mostly defined by low-frequency phonons according to the Bose-Einstein distribution. For such low temperatures, very long mfp's are observed. Increasing $T$ brings the phonon mfp population to shorter lengths characterized by an increased number of collisions. The mfp distribution shape clearly depends on the model used to model phonon lifetimes. In the case of the Holland model, where a cutoff frequency $\omega_{12}$ is defined to describe transverse Umklapp processes that arise at high temperatures, it induces the occurrence of two peaks in the mfp distribution at $T \geqslant 500 \mathrm{~K}$.

In the latter considered case (Fig. 1), most of the phonons undergo collisions for $5<\mathrm{mfp}<100 \mathrm{~nm}(90 \%)$ and $5 \%$ of them have mfp's up to $2 \mu \mathrm{m}$. A more detailed analysis also shows that low-frequency LA modes and TA modes on the edge of the Brillouin zone contribute more efficiently to long mfp's. This is naturally related to the chosen phonon lifetime model used (Holland's model in this case). Similar tendencies are observed with the Debye-Callaway model. Other illustrations, at different temperatures that emphasize the transition between those two limits, are proposed in the Supplemental Material [12] for both phonon mfp distributions and heat flux autocorrelations.

\section{Autocorrelation calculations}

For each of the computed phonon trajectories (particle $i$ ), we also store time variations of the frequency, polarization branch index, and group velocity. We can thus determine the instantaneous heat flux of particle $i$ at time $t$ as

$$
\mathbf{q}(t, i)=\frac{1}{V} p(t, i) \hbar \omega(t, i) \mathbf{v}(t, i) \mathcal{W}(t),
$$

with $p$ the polarization degeneracy index, $V$ the control volume, and $\mathcal{W}(t)$ a weighting factor defined as the energy ratio of the theoretical phonon energy in the considered system at $T$ according to the Bose-Einstein distribution to the sampled phonon energy. Thus, $\mathcal{W}(t)$ reads

$$
\mathcal{W}(t)=\frac{E^{t h}(T)}{E^{\operatorname{sampl}}(t)},
$$

where

$$
E^{t h}(T)=\int_{\omega=0}^{\omega_{\max }} \sum_{p=L A, T A} \frac{1}{\left[\exp \left(\hbar \omega / k_{B} T\right)-1\right]} D(\omega) p \hbar \omega d \omega,
$$

with $D(\omega)=K^{2} /\left(2 \pi^{2} v_{g}\right)$ the phonon density of state, and $p$ the polarization degeneracy ( $p=1$ for LA and $p=2$ for TA branches). Finally, the sampled energy reads

$$
E^{\mathrm{sampl}}(t)=\sum_{i=1}^{N_{p}} p(i) \hbar \omega(i)
$$

The proportionality factor $\mathcal{W}(t)$ is commonly used in MC modeling of the BTE, as the number of sampled particles in simulations is always much smaller than the actual number of phonons. Within this framework, instantaneous fluctuations of the heat flux around the equilibrium temperature are known and the Green-Kubo formalism can be applied.

Usually, the assessment of TC with the G-K formalism relies on equilibrium MD calculation of heat currents in an atomic system. The resulting expression of the thermal conductivity is a consequence of the fluctuation-dissipation theorem [14]; it reads

$$
k_{\alpha, \beta}=\frac{V}{k_{B} T^{2}} \int_{0}^{\infty}\left\langle q_{\alpha}(0) q_{\beta}(t)\right\rangle d t,
$$

where $\alpha$ and $\beta$ are the Cartesian coordinates of $\mathbf{q}\left(q_{x}, q_{y}\right.$, and $q_{z}$ ). Practically, for each time step, the heat flux carried per each "particle" is calculated and stored. The resulting heat flux history is split into $M=N_{t} / 10$ samples to compute discrete values of the heat flux autocorrelation (HFAC). In this work, we used the same formalism as detailed by Schelling et al. [5]:

$$
k_{x, y}=\frac{V}{k_{B} T^{2}} \frac{\delta t}{N_{p}} \sum_{p=1}^{N_{p}} \sum_{m=1}^{M} \frac{1}{N_{t}-m} \sum_{n=1}^{N_{t}-m} q_{x}(m+n) q_{y}(n) .
$$

The computed TCs are averaged on the number $N_{p}$ of launched particles. Time steps are in the range $1<\delta t<5$ ps. Longer time steps are usually required at low temperatures to follow long correlation times related to ballistic transport of energy carriers.

We thus can derive the components of the TC tensor. In Fig. 4 are plotted the HFACs and their integration according to Eq. (7) for $\mathrm{Si}$ at $300 \mathrm{~K}$ using computed phonon tracks and related values of instantaneous heat fluxes. In this particular case of $\mathrm{Si}$ at room temperature, some heat flux components remain correlated for more than $1 \mathrm{~ns}$. The latter constitute $3 \%$ of 

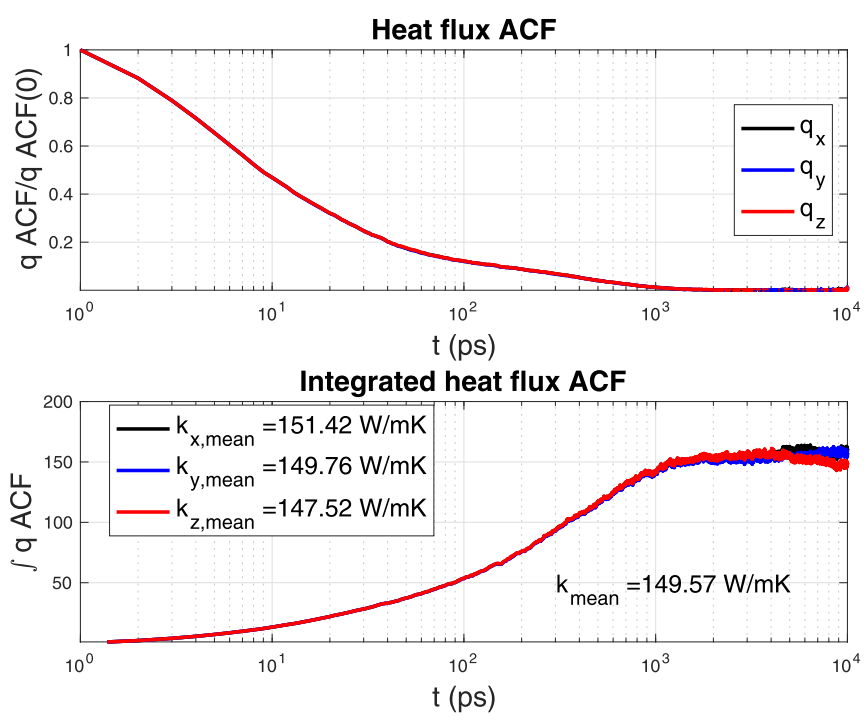

FIG. 4. Top: Heat flux autocorrelation for $x, y$, and $z$ components for $\mathrm{Si}$ at $300 \mathrm{~K}$ in the bulk. Bottom: Thermal conductivity components $k_{x x}, k_{y y}$, and $k_{z z}$. Five hundred particles were followed during 100000 time steps of 1 ps. Phonon lifetimes according to Holland's formalism [11].

the total number of energy carriers traveling in the Si domain. Integration of the HFAC for a duration of $10 \mathrm{~ns}$ shows that the mean TC reaches a constant value of $149.6 \mathrm{~W} \mathrm{~m}^{-1} \mathrm{~K}^{-1}$, in very good agreement with the experimental one. In addition, it can be seen that the three components of the TC tensor are equivalent. This was expected, as we consider here an isotropic material and a bulk system. It will be shown further that setting diffuse reflection for phonons that propagate in a bounded simulation domain will lead to a reduced TC in one or several directions.

As mentioned above, simulations where energy carriers have long mfp's require longer computation times to ensure a good calculation convergence. Conversely, at high temperatures, the calculation of thermal conductivity converges quickly. In this sense, the coupled MC-G-K equilibrium approach is interesting to describe the transport properties at high temperatures compared to the classical ETB resolutions, by the MC method, where a thermal gradient is applied [6]. Indeed, in the latter case, reaching the steady state necessary to establish the heat flow is much longer, which makes such computations time-consuming. Thus, in these cases, the MCG-K method is very efficient. Several illustrations of HFACs for the materials considered in this work are reported in the Supplemental Material [12].

\section{Convergence of the method}

Herein we report some details concerning simulation and, more specifically, convergence and accuracy of the method compared to more traditional approaches based on the MC solution of the BTE. Indeed, there are three main criteria that might affect calculation convergence: (i) $N_{p}$, the number of tracked particles; (ii) $N_{b}$, the number of frequency bins over which the phonon frequency domain is split; and (iii) $N_{t}$, the number of time steps used to reach convergence.

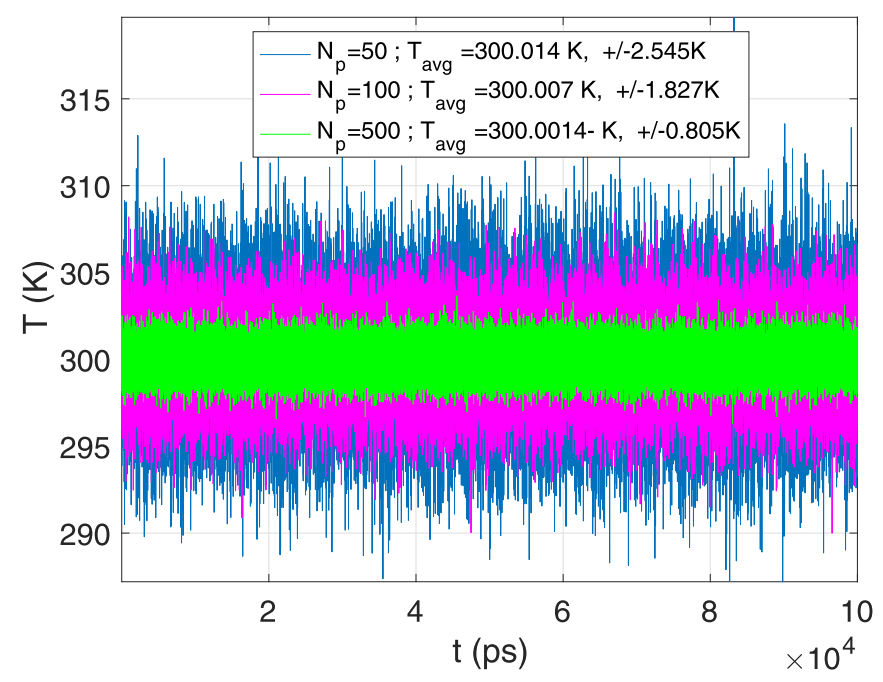

FIG. 5. Temperature fluctuations in the domain vs simulation time according to the number of sampled particles. $N_{p}=50,100$, and 500 .

Regarding the last criterion, $N_{t}$, the choice of this parameter is clearly dependent on the temperature and the nature of the material. $N_{t}$ must be sufficiently large so that the calculation of the autocorrelation of the flux is not biased. That is, over the time during which a phonon is "followed" we must ensure that there is no correlation after a finite number of iterations (here fixed at $N_{t} / 10$ ). Of course, if the material is a good conductor, we have to increase $N_{t}$. A similar consideration can be made at low temperatures when the transport is mostly "ballistic" and the phonons collide rarely. As mentioned previously, $N_{t}$ has been chosen between 100000 and 200000 time steps.

$N_{b}$ is the number of spectral bands used for the calculation; in this study the number was fixed at 500 over the range from 0 to $\omega_{\text {LAmax }}$. This choice corresponds to what is generally used in the classical MC approach to solve the Boltzmann equation (under the assumption of isotropic dispersion). Increasing the number of bands does not significantly improve the accuracy; if it is reduced to less than 100, there may be biases when sampling the carrier frequencies.

Finally, it turns out that the choice of $N_{p}$ is not decisive in the sense that the calculation can be conducted satisfactorily even with fewer particles (100 or even 50) and give a correct estimation of the thermal conductivity. On the other hand, we note a stronger amplitude of the temperature variation when $N_{p}$ is lowered (see Fig. 5). In practice, during the initial stage, the theoretical energy of the system, for a volume $V$ and temperature $T$, is shared between $N_{p}$ phonons. As phonons may collide, their frequency may change and, consequently, their energy and the resulting average temperature. In the frame of these calculations (which are supposed to be around an equilibrium temperature), using fewer particles could lead to larger instantaneous variations of the energy and thus to larger temperature variation around equilibrium. Multiplying the number of particles $N_{p}$ by 10 (from 50 to 500) divides the standard deviation by approximately 3 , i.e., from 2.545 to $0.805 \mathrm{~K}$. Here the standard deviation of the computed temperature scales with the square root of the sampled particles. The 
TABLE I. Temperature and thermal conductivity variations as a function of $N_{p}$, the number of sampled particles; the case of silicon at $300 \mathrm{~K}$ with the Holland model.

\begin{tabular}{lccc}
\hline \hline & \multicolumn{3}{c}{$N_{p}$} \\
\cline { 2 - 4 } & 50 & 100 & 500 \\
\hline$T_{\text {avg }}(\mathrm{K})$ & 300.014 & 300.007 & 300.001 \\
$\sigma_{T}(\mathrm{~K})$ & 2.545 & 1.827 & 0.805 \\
$k\left(\mathrm{~W} \mathrm{~m}^{-1} \mathrm{~K}^{-1}\right)$ & 150.62 & 143.44 & 149.57 \\
$\sigma_{k}\left(\mathrm{~W} \mathrm{~m}^{-1} \mathrm{~K}^{-1}\right)$ & 12.95 & 7.67 & 1.96 \\
\hline \hline
\end{tabular}

impact on the computed thermal conductivity is low regarding the average value but leads to larger uncertainties. Hereafter are reported in Table I the $T$ and $k$ average values and their related standard deviations.

\section{THERMAL CONDUCTIVITY CALCULATION WITH THE MG-G-K METHOD}

At this stage, we have shown that this new method combining $\mathrm{MC}$ phonon transport and the G-K formalism works to recover the value of bulk $\mathrm{Si} \mathrm{TC}$ at room temperature. To demonstrate that the approach remains efficient over a larger temperature range and for several materials, we performed calculations on almost 100 different configurations, i.e., changing the material $(\mathrm{Si}, \mathrm{Ge}, \mathrm{GaN}, \mathrm{C})$, the temperature $(80<T<600 \mathrm{~K})$, and the phonon lifetime model (Debye-Callaway or Holland). For each case, the TC tensor is computed for the bulk system and simulation results are compared to available experimental data. First, for the four considered materials, lifetime parameter sets were computed in the framework of the kinetic theory model using isotropic dispersion relations. Experimental TC values have been taken from the literature for $\mathrm{Si}$ and $\mathrm{Ge}$ [15], for $\mathrm{GaN}$ [16], and for $\mathrm{C}$ diamond [17].

We first report the $\mathrm{Si}$ and $\mathrm{Ge}$ results. In both Figs. 6 and 7 , red circles correspond to the material bulk thermal conductivity obtained by applying kinetic theory. For the sake of clarity, only data obtained with the Debye-Callaway model, using the kinetic theory, are reported (see Figs. 2-5 in the Supplemental Material [12]). These circles, which nicely match the experimental TC values, are the results of a phonon lifetime parameter adjustment. Depending on the theoretical model that is considered, impurity, normal, and Umklapp scattering mechanisms are characterized by analytic forms $[10,11]$ which allow us to reproduce the bulk TC over a broad temperature range. These parameters are inputs to $\mathrm{MC}$ simulations; they are provided in Tables 1-3 in the Supplemental Material [12]. Once the calculations are done, these parameters remain unchanged in MC-G-K calculations. For Si (Fig. 6), the matching between experiments and simulations is satisfactory for low and high TC values whichever phonon lifetime model is chosen. For Ge (Fig. 7) the agreement is globally satisfactory even if MC-G-K simulations tend to underestimate large $\mathrm{TC}$ values. The latter correspond to temperatures in the range of 50 to $80 \mathrm{~K}$, where impurity and disorder scattering are significant as well as normal scattering. According to Morelli et al. [10], adjustment of lifetime param-

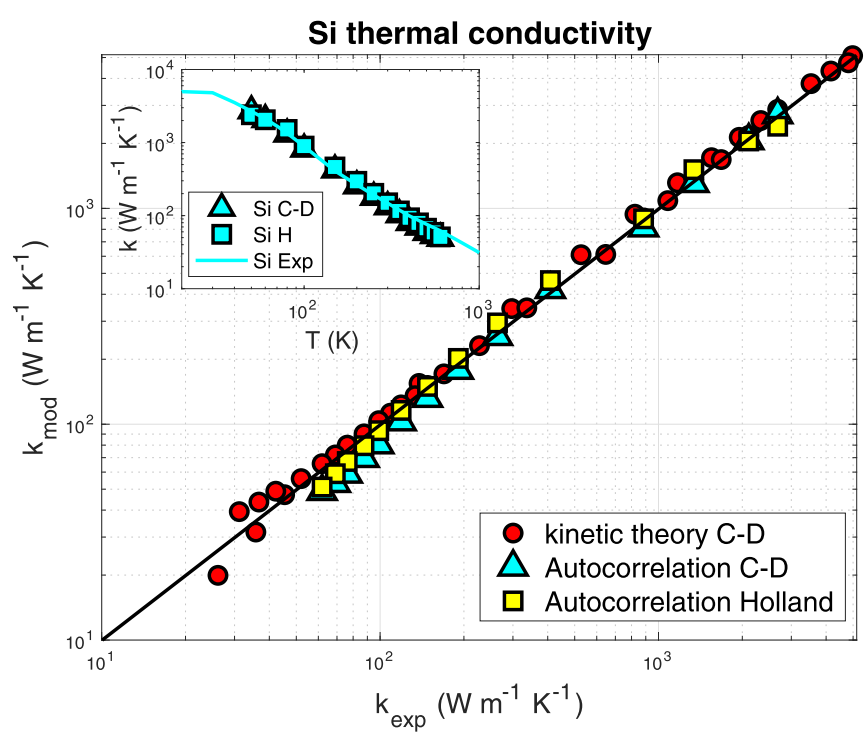

FIG. 6. Computed TCs vs their experimental counterparts for Si [15]. Inset: $k$ vs $T$.

eters in this temperature range can be complex. In addition, it is well known that the Ge TC is very sensitive to the doping level, which may also induce bias between experimental and numerical values.

The last figures, Figs. 8 and 9, are related to $\mathrm{GaN}$ and $\mathrm{C}$ in the diamond phase, respectively. As in the previous case, $\mathrm{GaN}$ calculations are in good agreement with experiments except at low $T$ (50 and $60 \mathrm{~K})$. The same comments apply to explain such little discrepancies. Furthermore, there is a quite large dispersion of the bulk TCs experimentally obtained in the literature for temperatures below $80 \mathrm{~K}$ [16]. More interestingly, in the case of diamond, the trends are a bit different. First, low TC values somehow underestimate the experimental ones, especially using the Holland model.

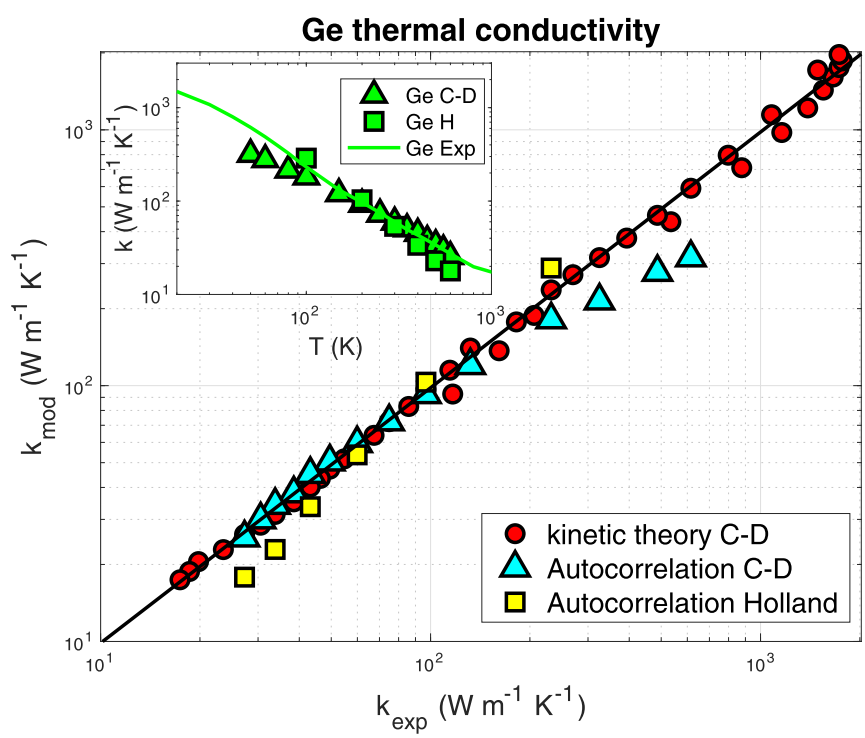

FIG. 7. Computed TCs vs their experimental counterparts for Ge [15]. Inset: $k$ vs $T$. 


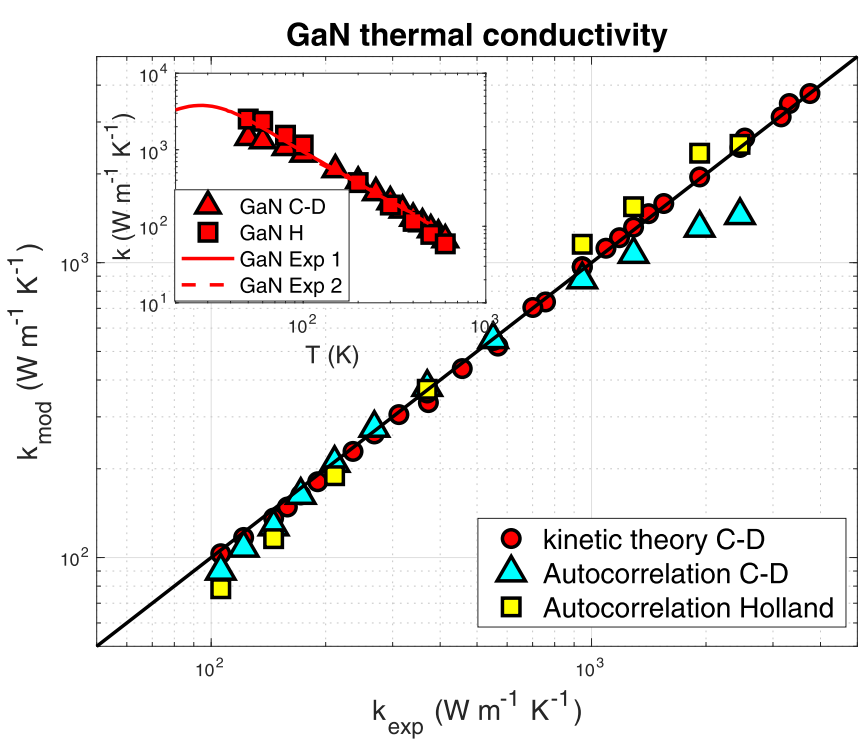

FIG. 8. Computed TCs vs their experimental counterparts for GaN [16]. Inset: $k$ vs $T$.

This also occurs with the kinetic model used to perform the parametrization of lifetimes. Such behavior can be explained by the limit of the theoretical model to predict the thermal conductivity in highly conductive material at high temperatures. As shown in the Supplemental Material [12], both models more or less failed to correctly predict the diamond TC for temperatures higher than $500 \mathrm{~K}$. In the latter case, adjustment of the Umklapp lifetime in this weakly anharmonic material is complicated, in particular, with the Holland model. On the other hand, for high TCs (low-temperature case), the MC simulation tends to strongly overestimate the experimental values. The origin of this error comes from the "infinite size" of the simulated system and the large mean free paths of phonons at these temperatures. Indeed, trajectory tracking shows that

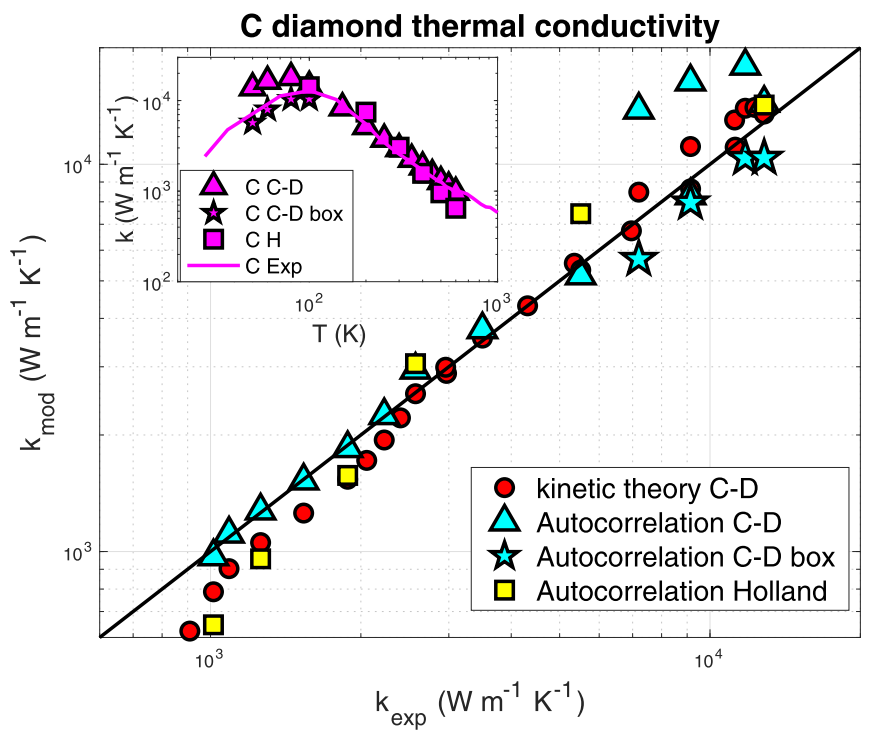

FIG. 9. Computed TCs vs their experimental counterparts for C diamond [17]. Inset: $k$ vs $T$. over the duration of the simulation (200 000 time steps of $5 \mathrm{ps,}$ i.e., $1 \mu \mathrm{m} \mathrm{s}$ ) the energy carriers traveled several millimeters, which is comparable to the size of the samples used for the measurements [17]. Hence, to take into account a possible scattering effect on the boundaries, the computational domain was limited to $1 \mathrm{~mm}$ per side and the phonons reaching these limits were diffusely reflected. Thus, an additional scattering mechanism has been added to the MC model and the corresponding thermal conductivity values (light-blue stars in Fig. 9) are more consistent with the experiments.

The ability of the MC-G-K model to take into account physical boundaries induced by the system geometry opens the way to new calculations of thermal transport properties in nanostructures such as thin films, nanowires, and quantum dots. In order to model these devices, careful attention must be paid to the design of phonon reflection and scattering at the boundaries. For example, setting diffuse reflection conditions on the edge of the simulation box or on engineered pores allows nanofilm, nanowires, and nanoporous samples to be mimicked $[6,18,19]$. Other kinds of nanostructures like devices with interfaces (superlattices, films with nanoinclusions, etc.) could also be investigated with a suitable model for phonon transmission or reflection across the interface. In addition, we also note that the method can make use of the full band model approach regarding the definition of phonon dispersion properties and phonon lifetimes to eliminate semianalytic models. We have used DFT dispersions and lifetimes in the classic MC solution of the BTE [8]. Implementation of the latter is not straightforward and the simulation domain should be subdivided into smaller cells in order to evaluate local temperature fluctuations and heat flux variations. Such considerations are currently under study and will be presented in future works.

\section{CONCLUSIONS}

To conclude, in this paper, we have presented an original approach to combine the advantages of the Green-Kubo theory for the calculation of transport properties with the Monte Carlo method, particularly well adapted to the solution of the Boltzmann transport equation. According to the considered system, this new method can be more efficient than classic solution of the BTE, especially when the phonon transport is far from the ballistic regime (high $T$, large system). Another asset of the method is its ability to work at a constant temperature and to give, in a single calculation, the TC tensor. On the other hand, the method is less efficient when ballistic transport dominates, as heat fluxes remain correlated over long durations, which implies the performance of longer computations. This type of approach had never been proposed for semiconductor materials. It is generalizable to other materials and could be coupled to other models. We have already demonstrated that the statistical tool lends itself well to the calculation of the thermal conductivity of complex materials whose phonon properties were derived from $a b$ initio calculations. Finally, the potential to control the nature of the boundaries of the studied system and, in particular, to define a collision model (specular, diffuse, etc.) opens vast possibilities for this method in the field of nanostructured materials. 


\section{ACKNOWLEDGMENTS}

The authors thank Dr. Laurent Chaput for fruitful discussions. This paper contains the results obtained in the framework of the project "Spider-Man" (ANR-18-CE42-0006).
This work was performed using HPC resources from GENCITGCC and GENCI-IDRIS (Grant No. 2020-A0080907186), in addition HPC resources were partially provided by the EXPLOR centre hosted by the University de Lorraine.
[1] G. Pernot, M. Stoffel, I. Savic, F. Pezzoli, P. Chen, G. Savelli, A. Jacquot, J. Schumann, U. Denker, I. Mönch, Ch. Deneke, O. G. Schmidt, J. M. Rampnoux, S. Wang, M. Plissonier, A. Rastelli, S. Dilhaire, and N. Mingo, Precise control of thermal conductivity at the nanoscale through individual phonon-scattering barriers, Nat. Mater. 9, 491 (2010).

[2] D. A Broido, M. Malorny, G. Birner, N. Mingo, and D. A. Stewart, Intrinsic lattice thermal conductivity of semiconductors from first principles, Appl. Phys. Lett. 91, 231922 (2007).

[3] A. Togo, L. Chaput, and I. Tanaka, Distributions of phonon lifetimes in Brillouin zones, Phys. Rev. B 91, 094306 (2015).

[4] S. Volz and G. Chen, Molecular-dynamics simulation of thermal conductivity of silicon crystals, Phys. Rev. B 61, 2651 (2000).

[5] P. K. Schelling, S. R. Phillpot, and P. Keblinski, Comparison of atomic-level simulation methods for computing thermal conductivity, Phys. Rev. B 65, 144306 (2002).

[6] D. Lacroix, K. Joulain, and D. Lemonnier, Monte Carlo transient phonon transport in silicon and germanium at nanoscales, Phys. Rev. B 72, 064305 (2005).

[7] C. Mangold, S. Chen, G. Barbalinardo, J. Behler, P. Pochet, K. Termentzidis, Y. Han, L. Chaput, D. Lacroix, and D. Donadio, Transferability of neural network potentials for varying stoichiometry: Phonons and thermal conductivity of MnxGey compounds, J. Appl. Phys. 127, 244901 (2020).

[8] L. Chaput, J. Larroque, P. Dollfus, J. Saint-Martin, and D. Lacroix, Ab initio based calculations of the thermal conductivity at the micron scale, Appl. Phys. Lett. 112, 033104 (2018).

[9] D. Bruno, Direct simulation Monte Carlo simulation of thermal fluctuations in gases, Phys. Fluids 31, 047105 (2019).

[10] D. T. Morelli, J. P. Heremans, and G. A. Slack, Estimation of the isotope effect on the lattice thermal conductivity of group IV and group III-V semiconductors, Phys. Rev. B 66, 195304 (2002).

[11] M. G. Holland, Analysis of lattice thermal conductivity, Phys. Rev. 132, 2461 (1963).

[12] See Supplemental Material at http://link.aps.org/supplemental/ 10.1103/PhysRevB.104.165202 for phonon dispersion properties, Debye-Callaway and Holland lifetime models, mfp and autocorrelation calculations for the four materials at various temperatures, which includes Refs. [20-22].

[13] B. Vermeersch, A. M. S. Mohammed, G. Pernot, Y. R. Koh, and A. Shakouri, Superdiffusive heat conduction in semiconductor alloys. II. Truncated Lévy formalism for experimental analysis, Phys. Rev. B 91, 085203 (2015).

[14] M. Toda, R. Kubo, N. Saito, and N. Hashitsume, Statistical Physics II: Nonequilibrium Statistical Mechanics (Springer, Berlin, 2012).

[15] C. J. Glassbrenner and G. A. Slack, Thermal conductivity of silicon and germanium from $3 \mathrm{~K}$ to the melting point, Phys. Rev. 134, A1058 (1964).

[16] A. V. Inyushkin, A. N. Taldenkov, D. A. Chernodubov, V. V. Voronenkov, and Y. G. Shreter, High thermal conductivity of bulk GaN single crystal: An accurate experimental determination, JETP Lett. 112, 106 (2020).

[17] J. R. Olson, R. O. Pohl, J. W. Vandersande, A. Zoltan, T. R. Anthony, and W. F. Banholzer, Thermal conductivity of diamond between 170 and $1200 \mathrm{~K}$ and the isotope effect, Phys. Rev. B 47, 14850 (1993).

[18] D. Lacroix, K. Joulain, D. Terris, and D. Lemonnier, Monte Carlo simulation of phonon confinement in silicon nanostructures: Application to the determination of the thermal conductivity of silicon nanowires, Appl. Phys. Lett. 89, 103104 (2006).

[19] V. Jean, S. Fumeron, K. Termentzidis, S. Tutashkonko, and D. Lacroix, Monte Carlo simulations of phonon transport in nanoporous silicon and germanium, J. Appl. Phys. 115, 024304 (2014).

[20] E. Pop, W. Dutton, and K. E. Goodson, Monte Carlo simulation of joule heating in bulk and strained silicon, Appl. Phys. Lett. 86, 082101 (2005).

[21] J. Callaway, Model for lattice thermal conductivity at low temperatures, Phys. Rev. 113, 1046 (1959).

[22] M. Asen-Palmer, K. Bartkowski, E. Gmelin, M. Cardona, A. P. Zhernov, A. V. Inyushkin, A. Taldenkov, V. I. Ozhogin, K. M. Itoh, and E. E. Haller, Thermal conductivity of germanium crystals with different isotopic compositions, Phys. Rev. B 56, 9431 (1997). 